\title{
Effects of Focal Vibration and Robotic Assistive Therapy on Upper Limb Spasticity in incomplete Spinal Cord Injury
}

\author{
Tijana Jevtic Vojinovic ${ }^{1,2}$, Emma Linley ${ }^{3}$, Aleksandar Zivanovic ${ }^{1}$ and Rui C.V. Loureiro ${ }^{2}$
}

\begin{abstract}
Vibration stimulation seems to be an affordable easy-to-use rehabilitation tool. Focal muscle vibration (FV) has potential to reduce spasticity and enhance muscle strength and performance. Combined with robotic assisted movement therapy, the rehabilitation can benefit from improvement of more than one aspect. For example, FV could firstly decrease abnormally increased muscle tone and joint rigidity by tackling volitional control for easier robotic movement exercise. Exactly this approach is evaluated within a clinical trial presented in this paper. FV were applied to relaxed spastic wrist flexor and extensor muscles for $15 \mathrm{~min}$. Subsequently, the wrist was engaged in a robotic-assisted game-playing. Results from two cases who completed the trial showed short-term decrease in wrist stiffness as assessed by clinical spasticity measurement Modified Ashworth Scale (MAS). Active range of motion (AROM) and engineering joint stiffness (JS) measurements were estimated using a robotic apparatus and the results complemented previous observations. The AROM increased and JS decreased for both cases when compared at the beginning and at the end of each interventional session. These results are a part of an ongoing clinical trial but show promise for reducing repercussions of spasticity in incomplete spinal cord injury.
\end{abstract}

\section{BACKGROUND}

Spasticity is a common repercussion after spinal cord injury (67\% to $78 \%$ ) contributing to the development of contractures, pressure ulcers, infections and degradation in posture, sitting and the ability of to use wheelchairs [1]. Vibration stimulation is one of the promising treatments for spasticity, with the least side effects compared to pharmaceutical agents [2]. Given that vibration modalities (whole body, segmental or focal) have similar modulation mechanisms regardless of the type, the low-cost and ease-of-use puts focal vibration (FV) at the top of the list as a preferred choice [3]. Furthermore, unlike for focal vibrations, the literature provides inconsistent effectiveness of whole body vibrations and segmental vibrations against spasticity [4].

A salient point to arise from our previous research with healthy participants is that FV applied to a relaxed muscle have potential to enhance muscle power by tapping into

This work was partially supported by Rehabilitation Technologies Supporting Clinical and Self-management of Spasticity project (RESPONSS) funded by Leslie Trust Fund

${ }^{1}$ Tijana Jevtic Vojinovic and Rui C.V. Loureiro are with Aspire CREATe - Centre for Rehabilitation Engineering and Assistive Technology, Department of Orthopaedics and Musculoskeletal Science, University College London, United Kingdom t.jevtic@ucl.ac.uk, r.loureiro@ucl.ac.uk

${ }^{2}$ Tijana Jevtic Vojinovic and Aleksandar Zivanovic are with Department of Design Engineering and Mathematics, Faculty of Science and Technology, Middlesex University London, United Kingdom a.zivanovicemdx.ac.uk

${ }^{2}$ Emma Linley is with Occupational Therapy, Royal National Orthopaedic Hospital, Stanmore, United Kingdom emma. I inley@nhs . net cortical responses, spinal reflex mechanisms and muscle activation patterns [5], [6]. This could be of importance in spinal cord injury (SCI) rehabilitation to increase motor abilities of the residual movements. Calabro et al applied FV to a tendon and Casale et al to several arm and shoulder muscles during robotic-aided exercise for people with stroke and observed effectiveness in decreasing spasticity and improvement in volitional kinematic activities [7], [8]. On the other hand, Backus et al followed a similar approach for people with spinal cord injury with focal tendon vibration and reported little or no change in spasticity [9].

The ongoing clinical trial presented in this paper investigates the effects of focal muscle vibration accompanied by a robotic-assisted therapy on several aspects of spasticity manifestation. They are outlined in section II-B. The apparatus described in our previous work is used to measure volitional abilities, range of motion and joint stiffness, and equally provide robotic-assisted mobility therapy. Additionally, clinical measure of spasticity (Modified Ashworth Scale or MAS) is employed to further affirm results [10].

\section{INTRODUCTION}

\section{A. VIBROfocus hardware}

The VIBROfocus system [10] is designed for delivering therapy and measuring outcomes of a wrist joint affected by abnormally increased muscle tone in wrist flexors and/or extensor muscles. Therapy delivery is comprised of two approaches: focal vibration stimulation and repetitive roboticassisted movement therapy. In addition to clinical measurement of spasticity (Modified Ashworth Scale), the robotic apparatus allows for the engineering outcome evaluation (active and passive range of motion, joint stiffness and a range of kinematic parameters associated with roboticassisted therapy). By need, different electrophysiological recordings can be included.

FV are delivered using two encapsulated vibration motors with eccentric mass positioned on flexor and extensor muscles of the forearm. The contact surface of the vibration motors should not exceed the surface of the targeted muscle in any direction therefore limiting vibration exposure to the neighbouring tissues.

The VIBROfocus apparatus mechanical and kinematical design include a cable driven power transmission of a capstan from a back-drivable motor. The hand is positioned on top of the capstan in midsuppination between two force measurement plates. The plates are used to flatten curled hand (due to spasticity) and also monitor and record force produced by the hand in flexion and extension. This information is then 
used in control strategies detailed in section. Centre of the dorsal part of the ulnar side of the wrist is positioned in the centre of the rotation of the capstan. Together with the fixation of the palm between plates, this positioning limits wrist movement to only 2 directions: flexion and extension. The elbow is comfortably fixed in a resting bed allowing the forearm to float between the resting bed and the capstan. All electrical and mechanical components accompanying the apparatus are safely stored inside a wooden box above which the capstan and the elbow resting bed are situated.

\section{B. VIBROfocus software}

The VIBROfocus apparatus couples therapy delivery with online and offline outcome monitoring. The software is devised to manage data collection, movement control and safety assessment. A simple user interface accommodates easy selection and execution of a desired mode of operation. Data collection and watchdog are operating independently in the background, signalling to the operator only when there is a warning or an error.

As a part of the monitoring and assessment, several engineering measures are included which can evaluate wrist's stiffness and movement abilities. The measure presented in this paper are:

- Joint Passive Range of Motion (PROM) - furtherest position within a passive movement of the wrist joint into flexion and extension with no effort from the user.

- Joint Active Range of Motion (AROM) - furtherest position of user's ability to volitionally move the wrist joint into flexion and extension.

- Joint Stiffness (JS) - quantified by evaluating the joint force resistance against joint movements performed by the apparatus within PROM during constant velocities. Equation of a joint stiffness $k_{m}$ is given by (1):

$$
k_{m}=\frac{M}{\Theta}=\frac{F_{\max } \cdot 0.01}{\Theta}\left[\frac{N \cdot m}{d e g}\right]
$$

where $\mathrm{M}=\mathrm{F} \cdot \mathrm{d}$ is a torque, $\mathrm{F}$ maximal force, $\mathrm{d}$ distance from the centre of rotation and $\Theta$ is an angle of the corresponding force.

Robot-assisted therapy is achieved by engaging a user in a simple game playing: the PONG game. The user can control a paddle in the game by moving the wrist. The playing field can be mapped to different wrist ranges of motion to facilitate different spasticity levels and volitional abilities. For the same reason, the game can be played in one of three modes: 1) active (i.e. fully volitional), 2) active-assisted or 3) passive (i.e. no effort from the user). Active-assisted mode provides the user with time to initiate the movement but is assisted if they are not able to complete the movement in time to score a point. The game's level of difficulty can be adjusted to serve individual abilities and progress.

Control strategies depend on the mode of operation. The friction compensation controller is used when the user is asked to volitionally move the hand to a specific location (e.g. active range of motion, active or active-assisted game playing). The friction controller is active for a short period of time to overcome the initial friction due inertial of the mobile parts of the apparatus. The position controller is selected when the wrist needs to reach a certain position without the effort from the user (e.g. passive range of motion, passive or active-assisted game playing). Joint stiffness measurement is adapted position controller where the wrist is moved between limits of the passive range of motion under predetermined velocities.

Any movement within the apparatus is continuously monitored to ensure safety and comfort of the user. Apparatus benefits from both hardware and software hard-stops positioned on the far ranges of able-bodied range of motion. Sensors integrated within the system monitor slipping, velocities and optimal operation.

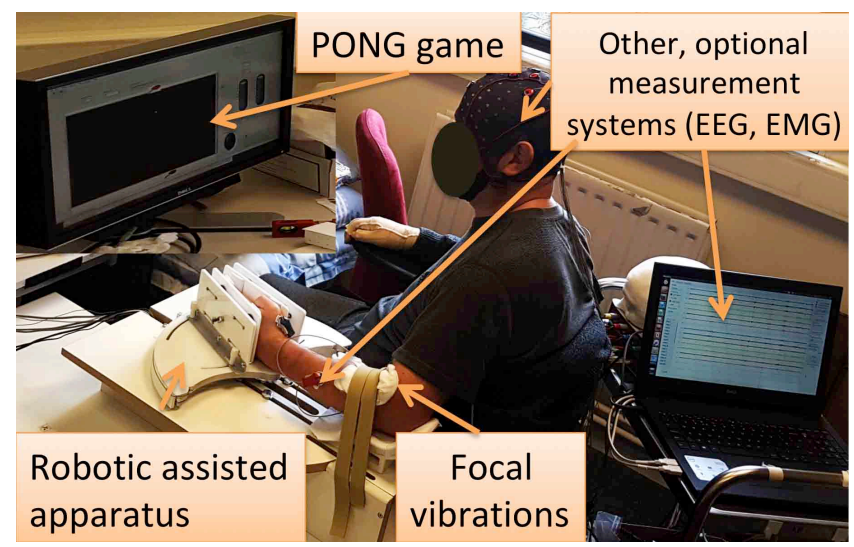

Fig. 1. General view of VIBROfocus system in use. User is seated in their wheelchair, in front of the computer screen while the forearm, wrist and the hand are positioned in the apparatus. Onscreen is the representation of a robotic-assisted therapy, the PONG game.

\section{Methodology}

\section{A. Ongoing Pilot Study}

The ongoing clinical trial presented in this paper was carried out to evaluate effects of focal vibrations coupled with robotic-assisted therapy on spasticity repercussions as a consequence of an incomplete spinal cord injury. It was designed as a pilot single case study assessing progress of each case as compared to the initial assessment, between specific times during each session and in-between sessions. The study was reviewed by NHS Health Research Authority Bromley Research Ethics Committee who raised no objection on ethical grounds and allowed the project to proceed.

\section{B. Participants}

Participants were recruited from the London Spinal Cord Injury Centre within the Royal National Orthopaedic Hospital NHS Trust, Stanmore. The preliminary results from two participants who completed the study are presented in this paper. Table I shows a demographic summary of the participants. The inclusion criteria considered Spinal Cord Injured level C1-6, AIS A-C (motor incomplete injury) and clinically diagnosed abnormal rigidity of the wrist (spasticity) at least Modified Ashworth Scale 1+. 
TABLE I

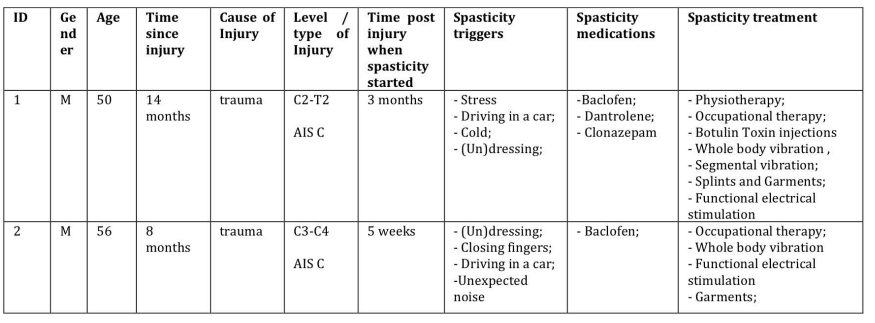

TABLE II

PROTOCOL STEPS

\begin{tabular}{c|c}
\hline 1 & Spasticity and Pain Assessment Questionnaire \\
\hline 2 & Modified Ashworth Scale \\
\hline 3 & Set up the wrist \\
\hline 4 & Set up the sensors \\
\hline 5 & Measure Active Range of Motion (AROM) \\
\hline 6 & Measure Passive Range of Motion (PROM) \\
\hline 7 & Measure Joint Stiffness (JS) \\
\hline 8 & Set up the vibration \\
\hline 9 & Apply focal vibrations \\
\hline 10 & Remove vibration motors \\
\hline 11 & Measure Active Range of Motion (AROM) \\
\hline 12 & Measure Passive Range of Motion (PROM) \\
\hline 13 & Measure Joint Stiffness (JS) \\
\hline 14 & Play a PONG game \\
\hline 15 & Measure Active Range of Motion (AROM) \\
\hline 16 & Measure Passive Range of Motion (PROM) \\
\hline 17 & Measure Joint Stiffness (JS) \\
\hline 18 & Take off the sensors \\
\hline 19 & Remove arm from the apparatus \\
\hline 20 & Modified Ashworth Scale \\
\hline 21 & Spasticity and Pain Assessment Questionnaire \\
\hline \multicolumn{2}{|c}{}
\end{tabular}

\section{Experimental protocol}

Each participant took part in total of 6 intervention sessions dispersed in 2 consecutive weeks (i.e. 3 sessions per week) using setup as seen in Fig. 1. The decision for this exposure pattern is based on the experience with spasticity rehabilitation treatment and the minimum time needed to record expected changes [11]. Results reported in the literature are showing that such exposure to robot therapy is often necessary to observe significant cortical reorganisation with the damaged brain and improved kinematic features (e.g. limb synergies and task oriented movements) [12].

At the beginning of the study, an initial assessment of MAS was recorded. This was followed by 6 interventional sessions, during each MAS was measured at the beginning and at the end of each session to minimise perturbation and repositioning of forearm/hand into the device and potentially induce a stretch or trigger spasticity. However, as can be noted in the table II, AROM, PROM, and JS were measured three time during each interventional session.

During each session, firstly, measurement of AROM was performed to capture volitional abilities participant might have. This is followed by PROM measured by a team member manually and slowly moving the capstan within able-bodied full range of motion. Limits to the range were recorded at the moment when the assessor started to felt an increase in resistance to movement. The justification for this type of measurement, aside from its similarity to performing a MAS, was to establish joint's limits to be set for the upcoming joint stiffness measurement. Joint stiffness was measured three times for three different velocities $8 \% \mathrm{sec}$, $44 \% \mathrm{sec}$ and $80 \% \mathrm{sec}$. The lowest and the fastest velocities were chosen as the most comfortable ones with minimum acceleration time and maximum constant velocity time during movements. This is to avoid potential jerks and estimate joint's behaviour during slow and fast movements. Velocity of $44 \% \mathrm{sec}$ is selected as a midrange velocity. The joint stiffness measurements were always performed in the same order, starting with the $8 \%$ sec and finishing with $80 \% \mathrm{sec}$ because fast movements are known spasticity triggers [2]. Participants were advised to inform the assessor if anytime during these measurement any discomfort, stiffening, spasms or other uncomfortable event occurred. Measurements of AROM, PROM and JS were performed at the each session's beginning (i.e. before vibration), after vibration stimulation, and at the end (i.e. after the game), always in the same order.

Focal vibrations were applied on the anterior and the posterior aspect of the forearm, approximately $5-7 \mathrm{~cm}$ from the elbow to cover muscle bellies for the majority of the wrist extensor and flexor muscles. Stimulation lasted 15 minutes at $75 \mathrm{~Hz}$ frequency and $0.4 \mathrm{~mm}$ amplitude. The game was played until participant would report tiredness, fatigue, or play time would exceed 20 minutes. Nonetheless, participants were encouraged to play the game as long as playing would imply enjoying and not any discomfort.

\section{Data Analysis}

PROM and AROM were programmed to capture both motion leading to the maximal angles of ROMs and maximal values in angles. Joint stiffness needed further processing as per previously stated equation (1). Programs controlling tasks execution, measuring and recording the data were written and executed in LabView environment (National Instruments). Recorded data was processes in MATLAB (The MathWorks, Inc.).

\section{RESULTS}

The results will be presented for each case independently.

\section{A. Case 1}

According to the initial MAS, demographic and spasticity manifestation assessment, this participant was classified as moderate to severe case of spasticity. The results for MAS measured at the beginning (before muscle focal vibration) and at the end (after game) of each interventional session are presented in figure 2. The gradual decrease in MAS at the beginning (red bar) of each session and compared between sessions can be observed for the extension (upper set of bars) but not for flexion (lower set of bars). Nonetheless, a contrast in score between session beginning (red bars) and end 
(subsequent purple bars) stipulates short term effectiveness of this multimodal approach.

A full passive range of motion (PROM $>110$ degrees) was observed during each measurement for every session. Case 1 had a neutral wrist position approximately in half flexion and was able to move the wrist in respect to that position. After the game in session 1, 2 and 5 case 1 was able to move wrist into extension as calculated from the neutral mid-supination position. Active range of motion, presented in figure 3 seems to improve after every muscle focal vibration stimulation during each session (comparison between joined red and black bars). Results suggest that both muscle focal vibration and robot-assisted exercise contribute to the improvement in the volitional ability to flex and extend the wrist joint between $30-60 \%$ (purple bars).

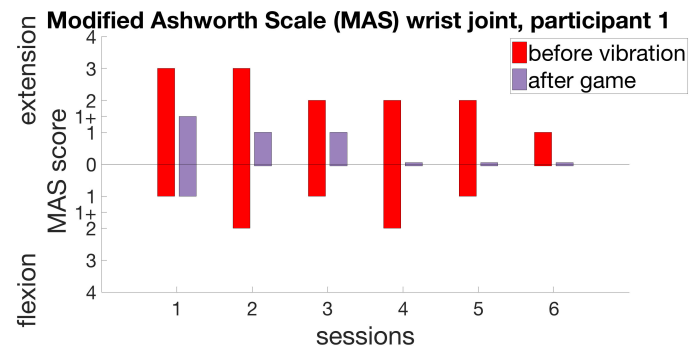

Fig. 2. Modified Ashworth Scale measured at two occasions during each sessions: red - before focal vibration, purple - after game.

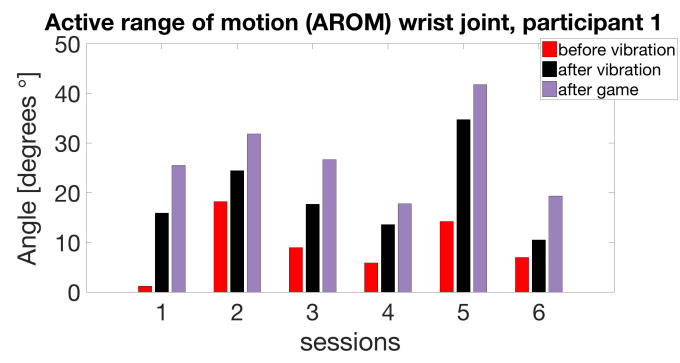

Fig. 3. Full active range of motion (AROM) measured at three occasion during each session: red - before focal vibration, black - after focal vibration, purple - after game.

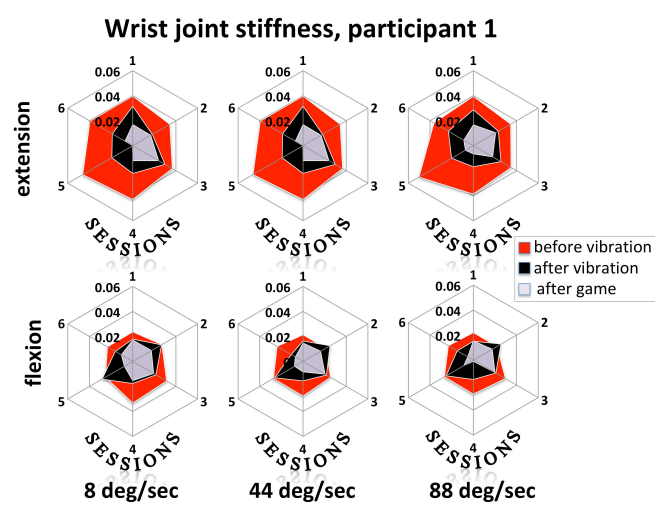

Fig. 4. Illustrative representation of wrist joint stiffness (JS) measured at three occasion during each session: red - before focal vibration, black - after focal vibration, purple - after game.
The wrist joint stiffness is presented in a rose plot for extension (up) and flexion (down) in figure 4 for three velocities in columns. This is an illustrative comparison, not a cyclic process. Extension joint stiffness seems more pronounced than flexion (upper plots). In extension, FV was able to reduce JS for every session. In flexion for faster movements (44deg/sec and 88deg/sec) during JS measurement in session 2 it can be observed that the joint stiffness slightly increased after the vibration stimulation (black area covering the red). Nonetheless it was reduced after game play (purple area).

\section{B. Case 2}

Initial assessment of MAS, demographic and spasticity manifestation assessment categorised the participant to mild to moderate spasticity levels. According to the MAS scores in figure 5 , session one is the only one where the score

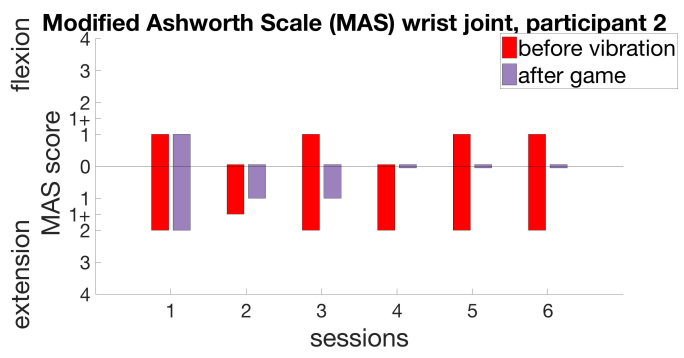

Fig. 5. Modified Ashworth Scale measured at two occasion during each session: red - before focal vibration, purple - after game.

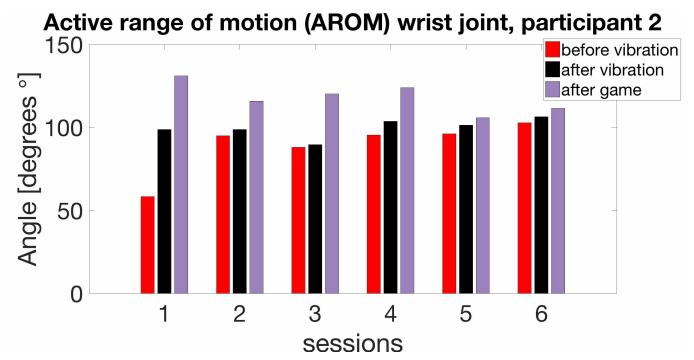

Fig. 6. Full active range of motion (AROM) measured at three occasion during each session: red - before focal vibration, black - after focal vibration, purple - after game.

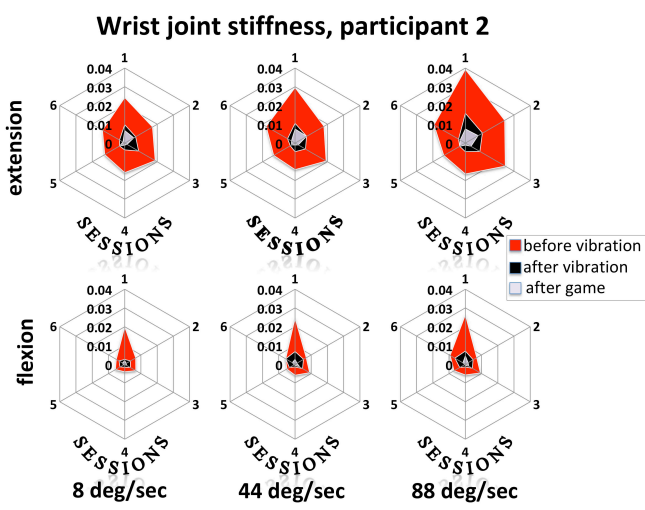

Fig. 7. Illustrative comparison of wrist joint stiffness (JS) measured at three occasion during each session: red - before focal vibration, black after focal vibration, purple - after game. 
did not change at the end of the session. Sessions 2 and 3 had some resistance in extension (bottom bars) while sessions 4, 5 and 6 had no spasticity observed while assessing MAS (contrast between red and purple bars). However, MAS scores at the beginning of each session did not change much in extension, but was diminished in sessions 3,4 and 5 after each interventional session. Flexion showed in top bars had very little spasticity (MAS 1 ) that recurred on sessions 1, 2, 5 and 6 but was nulled after the therapy.

A full passive range of motion (PROM $>110$ degrees) was observed during each measurement for every session. Case 2 had the ability to volitionally move the wrist in flexion and extension, as compared to the neutral mid-suppination position. This participant had the ability to perform high in the AROM measures seen in Fig. 6 during sessions 5 and 6 (red and purple bars), yet not much of a difference is observed in between session measures (purple bars).

The wrist joint stiffness is presented in rose plots in figure 7, upper row for extension, bottom for flexion and columns for different velocities. This figure doesn't present a cyclic process but a illustrative comparison of joint stiffness between sessions. High joint stiffness before vibrations (in red) was successfully reduced as compared after vibration (colour black) and after game (colour purple) in extension and flexion. While after the game there was some residual stiffness in extension (upper plots), for flexion it was almost diminished (bottom plots).

\section{Discussion}

Two cases with incomplete SCI are reported in this study with two different levels of upper limb spasticity severity. The main message that can be drawn here is that 15 minutes of high frequency $(75 \mathrm{~Hz})$ low amplitude $(0.4 \mathrm{~mm})$ muscle focal vibration (FV) followed by a robotic assisted movement therapy can decrease spasticity and induce functional improvements in volitional movements. Participants were very different in spasticity triggers and residual volitional control as a consequence of the difference in SCI injury. However, the consistency in spasticity reduction after six sessions, as measured with MAS and JS, was observed regardless to the level of injury. Both cases reported a change in subjective feeling of the hand and the wrist movements: quoting "feeling more relaxed and loose after the participation in the sessions". The carryover effects seem to depend on the usual triggers such as stress and dressing related activities. Nonetheless those correlated with the volitional control might have been minimised by this therapy. Both cases were excited with the functional improvements in the activities of the daily living such as handling a pen and writing and handling a glass.

Calabro et al reported that a similar approach applied to people with stroke can decrease spasticity and enhance movement kinematics [7]. They applied focal vibrations on the tendons whereas in this study FV was applied on the wrist joint flexor and extensor muscles of the forearm. The difference in location is very encouraging for the entire idea of FV spasticity aiding because the different application sites can induce different beneficial effects [13]. Having in mind that this study aimed at improvement of volitional functionality using focal muscle vibration, focal tendon stimulation could bring the aspect of proprioceptive feedback to the sensory deprived joints or even biofeedback to the user [5], [14].

Studies of the application of the FV to a spastic muscles of people with SCI, report reduction in spasticity measured by MAS [15], [11], complementing results of our study. Thus, Murillo et al observed a decrease in limb spasticity irrespective to the lesion being motor complete or incomplete after muscle FV [15]. This finding is important to assure that the type and the level of injury doesn't necessarily need to be regarded for the application of FV therapy, especially when our proposition to use FV coupled with robotic assisted therapy could advance the outcomes in movement therapy even after spasticity is diminished [16], [12]. Our study when completed, will also compare each treatment (i.e. vibrations and robotic-assisted therapy) to the control group (e.g. no therapy or conventional therapy) with similar and a range of demographic characteristics.

One might argue that JS might not be enough for the spasticity evaluation. This is one of the reasons why measurement of active and passive ROMs are proposed in this study in addition to JS [9], [14], [17], [18]. Interestingly enough, both cases had a full passive range of motion but different volitional ranges. This is somewhat expected due to severity of the spasticity not being extremely high (MAS 4). Nonetheless, the increase in volitional range of motion at the beginning and at the end of the study, due to FV stimulation, is consistent in both cases and in line with other ROM assessments [15]. Oh et al observed an grow in range of motion in the hemiplegic knee joint following 5 minutes of continuous calf muscle FV [19]. The results suggests the increase in ROM is sole contribution of FV. In our study, during each session, there is a surge in volitional angular displacement in extension and flexion, and therefore AROM, following both FV and robotic assister therapy. For case 1 the AROM persistence is highly dependent on the spasticity triggers such as stress level and activities of daily living. For example, the AROM measures during session 3 are lower than previous (sessions 1,2 and 3 performed in three consecutive days) possibly due to the 3 days difference between sessions 3 and 4 . Session 4 evoked a high increase in AROM which is lower again, possibly as a consequence of the reported pain in the ipsilateral shoulder. In contrast, case 2 had a consistent AROM with a few degrees in change. During qualitative assessment, both cases reported a strengthening of volitional abilities related to the wrist and hand movements. So, even though the increase in AROM is observed after FV, it seems reinforced after robotic assister therapy. Subjectiveness of spasticity to common triggers is something that should be addressed in future work, to assure robustness and persistence of the therapeutic effectiveness.

Literature imply promising results for recovery of volitional abilities of the hand in SCI, after a single session of robotic assister therapy [20]. Some of the mechanisms are inclusive of neuroplastic changes in the spinal and cortical 
level [21]. Neural plasticity in SCI consists of alterations or sprouting of new neural connections in order to reinforce and/or recreate control over activities, behaviours and sensory acquisition [22]. The repetition of the training over a period of time is essential for long term plasticity development. This is the reason why minimum of 6 sessions over 2 weeks is needed to observe the initial movement recovery patterns [23]. Moreover, this is in line with the timeline for the literature proposed FV spasticity recovery [8]. To accommodate the difference in residual movements, a game of PONG had three modes of playing: active (fully volitional), active assisted (movement is completed by the apparatus) and passive (movements are fully conducted by the apparatus). Case 1 played a game in active-assisted mode and case 2 in active mode. Celletti et al assured that combination of neurophysiologically-based rehabilitation techniques coupled with muscle FV have a high potential to recover hemiplegic impairments by altering spinal and supraspinal control of the movements [24]. The adaptiveness of a game (i.e. movement therapy) difficulty level over longer therapeutic period is hypothesised to be a way forward towards improving and perhaps even fully restoring functional volitional control. This claims should be supported by a larger randomised clinical trial with increased duration of a trial.

\section{CONCLUSION}

Focal muscle vibration stimulation of a relaxed muscles followed by a subsequent robotic assisted movement therapy can reduce repercussions of spasticity and joint stiffness in incomplete SCI. One of the advantages of this multimodal approach is tackling aspects of rigidity in muscles and joints in addition to volitional control and functional recovery. Even though more evidence is needed to support inclusion of this therapy into clinical practice, the results so far are shown to be very encouraging.

\section{ACKNOWLEDGMENT}

We wish to thank volunteers for their time and participation in the study.

\section{REFERENCES}

[1] F. M. Maynard, R. S. Karunas, and W. P. Waring, "Epidemiology of spasticity following traumatic spinal cord injury." Archives of Physical Medicine adn Rehabilitation, vol. 71, no. 8, pp. 566-569, Jul. 1990.

[2] J. Hsieh, D. L. Wolfe, A. F. Townson, and C. Short, "Spasticity following spinal cord injury," Spinal Cord Injury Rehabilitation Evidence, no. 3, 2012.

[3] D. G. Sayenko, K. Masani, M. Alizadeh-Meghrazi, M. R. Popovic, and B. C. Craven, "Acute effects of whole body vibration during passive standing on soleus H-reflex in subjects with and without spinal cord injury," Neuroscience Letters, vol. 482, no. 1, pp. 66-70, Sep. 2010.

[4] C. Brogårdh, U.-B. Flansbjer, and J. Lexell, "No Specific Effect of Whole-Body Vibration Training in Chronic Stroke: A Double-Blind Randomized Controlled Study," Archives of Physical Medicine and Rehabilitation, vol. 93, no. 2, pp. 253-258, Feb. 2012.

[5] T. Jevtic, A. Zivanovic, and R. C. V. Loureiro, "Focal vibro-tactile stimulation as a pre-conditioner to enhance muscle performance in robot-mediated neurorehabilitation," in 2015 IEEE International Conference on Rehabilitation Robotics (ICORR). IEEE, 2015, pp. 696701.

[6] T. Jevtic, A. Zivanovic, and R. Loureiro, "Cortical and Muscle Response to Focal Vibro-Tactile Stimuli," Converging Clinical and Engineering ..., vol. 2, pp. 893-897, 2016.
[7] R. S. Calabrò, A. Naro, M. Russo, D. Milardi, A. Leo, S. Filoni, A. Trinchera, and P. Bramanti, "Is two better than one? Muscle vibration plus robotic rehabilitation to improve upper limb spasticity and function: A pilot randomized controlled trial," PLoS ONE, vol. 12, no. 10 , pp. e0 $185936-20$, Oct. 2017.

[8] R. Casale, C. Damiani, R. Maestri, C. Fundarò, P. Chimento, and C. Foti, "Localized $100 \mathrm{~Hz}$ vibration improves function and reduces upper limb spasticity: a double-blind controlled study." European journal of physical and rehabilitation medicine, vol. 50, no. 5, pp. 495-504, Oct. 2014.

[9] D. Backus, P. Cordo, A. Gillott, C. Kandilakis, M. Mori, and A. M. Raslan, "Assisted Movement With Proprioceptive Stimulation Reduces Impairment and Restores Function in Incomplete Spinal Cord Injury," Archives of Physical Medicine adn Rehabilitation, vol. 95, no. 8, pp. 1447-1453, Aug. 2014.

[10] T. J. Vojinovic, A. Zivanovic, T. Carlson, and R. C. V. Loureiro, "VIBROfocus: Design of a focal vibro-tactile robotic-assistive system for spasticity rehabilitation," in 2017 International Conference on Rehabilitation Robotics (ICORR). IEEE, 2017, pp. 783-788.

[11] M. Etoom and A. Marchetti, "Effect of a Focal Muscle Vibration Above Triceps Brachii Muscle on Upper Limb Spasticity in a Patient with a Chronic Spinal Cord Injury: a Case Report," International Journal of Physiotherapy and Research, vol. 3, no. 4, pp. 1171-1174, Aug. 2015.

[12] I. Aprile, E. Di Sipio, M. Germanotta, C. Simbolotti, and L. Padua, "Muscle focal vibration in healthy subjects: evaluation of the effects on upper limb motor performance measured using a robotic device," European Journal of Applied Physiology, vol. 116, no. 4, pp. 729-737, Jan. 2016.

[13] G. Lee, Y. Cho, J. Beom, C. Chun, C. H. Kim, and B.-M. Oh, "Evaluating the Differential Electrophysiological Effects of the Focal Vibrator on the Tendon and Muscle Belly in Healthy People," Annals of Rehabilitation Medicine, vol. 38, no. 4, pp. 494-12, 2014.

[14] P. Cordo, H. Lutsep, L. Cordo, W. G. Wright, T. Cacciatore, and R. Skoss, "Assisted Movement With Enhanced Sensation (AMES): Coupling Motor and Sensory to Remediate Motor Deficits in Chronic Stroke Patients," Neurorehabilitation and Neural Repair, vol. 23, no. 1, pp. 67-77, Sep. 2008.

[15] N. Murillo, H. Kumru, J. Vidal-Samso, J. Benito, J. Medina, X. Navarro, and J. Valls-Sole, "Decrease of spasticity with muscle vibration in patients with spinal cord injury," Clinical Neurophysiology, vol. 122, no. 6, pp. 1183-1189, Jun. 2011.

[16] C. Tefertiller, B. Pharo, N. Evans, and P. Winchester, "Efficacy of rehabilitation robotics for walking training in neurological disorders: A review," The Journal of Rehabilitation Research and Development, vol. 48, no. 4, pp. 387-31, 2011.

[17] T. Noma, S. Matsumoto, S. Etoh, M. Shimodozono, and K. Kawahira, "Anti-spastic effects of the direct application of vibratory stimuli to the spastic muscles of hemiplegic limbs in post-stroke patients," Brain Injury, vol. 23, no. 7-8, pp. 623-631, Jul. 2009.

[18] S. Harini, V. S. Kumari, and K. Madhavi, "Effect of Low Frequency Vibratory Stimulation on Biceps Brachii Spasticity in Subjects with Hemiplegia," Indian Journal of Physiotherapy and Occupational Therapy - An International Journal, vol. 7, no. 4, pp. 192-6, 2013.

[19] J. Oh, J. H. Leigh, H. G. Seo, B. M. Oh, and J. Sangeun, "The effects of focal muscle vibration to reduce calf muscle spasticity in chronic stroke patients: Preliminary study," Journal of the Neurological Sciences, vol. 381, no. Supplement, p. 1134, Oct. 2017.

[20] L. Lucas, M. DiCicco, and Y. M. Mechatronics, "An EMG-controlled hand exoskeleton for natural pinching," Journal of Robotics and Mechatronics, vol. 16, no. 5, pp. 1-7, 2004.

[21] K. Fouad and W. Tetzlaff, "Rehabilitative training and plasticity following spinal cord injury," Experimental Neurology, vol. 235, no. 1, pp. 91-99, May 2012.

[22] Y. Ding, A. J. Kastin, and W. Pan, "Neural plasticity after spinal cord injury," Current pharmaceutical design, vol. 11, no. 11, pp. 14411450, 2005.

[23] V. W. Mark, E. Taub, and D. M. Morris, "Neuroplasticity and constraint-induced movement therapy." Europa medicophysica, vol. 42, no. 3, pp. 269-284, Sep. 2006.

[24] C. Celletti, E. Sinibaldi, F. Pierelli, G. Monari, and F. Camerota, "Focal mscle vibrations and progressive modular rebalancing with neurokinetic fascilitation," La Clinica terapeutica, vol. 168, no. 1, pp. e33-e36, Jan. 2017. 